\title{
M-ARY QUADRATURE AMPLITUDE MODULATION ORDER OPTIMIZATION FOR TERAHERTZ WIRELESS COMMUNICATIONS OVER DISPERSIVE CHANNELS
}

\author{
Karl Strecker ${ }^{1}$, Sabit Ekin $^{1}$, and John O’Hara ${ }^{1}$ \\ ${ }^{1}$ Department of Electrical and Computer Engineering, Oklahoma State University, Stillwater, OK, 74078 USA
}

NOTE: Corresponding author: John O’Hara, oharaj@okstate.edu

\begin{abstract}
Highly accurate atmospheric models, based on molecular resonance information contained within the HITRAN database, were used to simulate the propagation of high capacity single-carrier quadrature amplitude modulated signals through the atmosphere for various modulation orders. For high-bandwidth signals such as those considered in this work, group velocity dispersion caused by atmospheric gases distorts the modulated waveform, which may produce bit errors. This leads to stricter Signal-To-Noise Ratio requirements for error-free operation, and this effect is more pronounced in high-order modulation schemes. At the same time, high-order modulation schemes are more spectrally efficient, which reduces the bandwidth required to maintain a given data rate, and thus reduces the total group velocity dispersion in the link, resulting in less distortion and better performance. Our work with M-ary quadrature amplitude modulated signals shows that optimal selection of modulation order can minimize these conflicting effects, resulting in decreased error rate, and reducing the performance requirements placed on any equalizers, other dispersion-compensating technologies, or signal processing hardware.
\end{abstract}

Keywords - Atmospheric modeling, bit error rate, chromatic dispersion, millimeter wave communications, quadrature amplitude modulation, terahertz communications

\section{INTRODUCTION}

Wireless data rates have risen dramatically over the last decade, and are projected to continue to do so over the decade to come $[1,2]$. This growth has been fueled by demand, created by consumer expectations as well as new technologies such as virtual reality, high-definition video streaming, and (most significantly) the Internet of Things (IoT) $[3,4]$. This growth has been enabled by the development of devices capable of operating at progressively higher frequencies and bandwidths. Wireless systems operating at several gigahertz are commercially available off-the-shelf, and networks operating at several tens of gigahertz (millimeter wave) are just on the verge of becoming so. The inevitable next step is systems operating at sub-millimeter wavelengths, that is, hundreds of gigahertz [5]. This is frequently recognized as the beginning of the terahertz communication bands. These bands have been slow in development for many years, in part due to the challenge of atmospheric absorption and in part due to the technological difficulties arising from the fact that few devices are naturally active in these frequencies.

However, the so-called "terahertz gap" is beginning to close [6]. Recent progress in terahertz devices has resulted in hardware not only capable of producing and processing these high-speed signals, but also powerful enough to overcome the atmospheric attenuation, which is much more severe than at microwave frequencies. Over the last decade, several prototype terahertz communication systems have been demonstrated, operating in the hundreds of gigahertz, achieving communications over multi-kilometer distances.
For example, in 2010, Hirata et al. demonstrated a wireless link operating at $120 \mathrm{GHz}$, using Binary Phase Shift Keying (BPSK) that achieved an error-free data rate of 10 $\mathrm{Gb} / \mathrm{s}$ over $5 \mathrm{~km}$ [7]. In 2013, Takahashi et al. also demonstrated a $10-\mathrm{Gb} / \mathrm{s}$, error free link at $120 \mathrm{GHz}$, using Quadrature Phase Shift Keying (QPSK, or 4-QAM), over a distance of $170 \mathrm{~m}$ [8]. However, their calculations indicated the link could conceivably span up to $2 \mathrm{~km}$. The same year, another wireless link was demonstrated, this time at $140 \mathrm{GHz}$, using $16-\mathrm{QAM}$ to achieve $10 \mathrm{~Gb} / \mathrm{s}$ over $1.5 \mathrm{~km}$, with an error rate of $10^{-6}[9]$.

In 2017, another communication link centered at $94 \mathrm{GHz}$, using 8-QAM, achieved a data rate of $54 \mathrm{~Gb} / \mathrm{s}$, with an error rate of $3.8 \times 10^{-3}$, over $2.5 \mathrm{~km}$ [10]. Also in 2017, Kallfass et al. presented a review of their experimental work with point-to-point millimeter wave links which included an E-band link (between 60 and $90 \mathrm{GHz}$, carrier frequency not specified) and a $240 \mathrm{GHz}$ link [11]. The E-band link used QPSK, 8-QAM, and 16-QAMs, and achieved data rates in the range of $4 \mathrm{~Gb} / \mathrm{s}$ up to $21 \mathrm{~Gb} / \mathrm{s}$, over ranges between $4.1 \mathrm{~km}$ and $36.7 \mathrm{~km}$, under various weather con- ditions with error rates below $4.8 \times 10^{-3}$. The $240 \mathrm{GHz}$ link used QPSK modulation, and achieved $64 \mathrm{~Gb} / \mathrm{s}$ over $0.85 \mathrm{~km}$, with an error rate of $7.9 \times 10^{-5}$. Many different link configurations were investigated in the review, and the reader is referred to the work of Kalfass et al. for more detailed information [11].

Finally, Wu et al. also demonstrated a long-distance wireless communication system at $140 \mathrm{GHz}$ in 2017, which spanned $21 \mathrm{~km}$ and used 16-QAM to achieve $5 \mathrm{~Gb} / \mathrm{s}$ with effectively error-free operation (a bit-error rate below 
$10^{-12}$ ) [12]. The authors also estimated that their system could extend to span even farther ranges with the use of more advanced error-correction codes.

Collectively, these demonstrations indicate that longrange terahertz communication links are not only possible, but will likely be implemented commercially in the foreseeable future as the technology progresses. This technology would provide many benefits, since there are many situations in which the ability to rapidly establish a directive, wireless point-to-point link with a capacity of tens of $\mathrm{Gb} / \mathrm{s}$ would be highly attractive, including temporary installments during disaster recovery or wartime environments, at locations where trenching fiber is prohibitively expensive or time-consuming, or as a re- placement to upgrade microwave point-to-point backhaul links.

There are many design choices that must be considered when planning the construction of such a link $[13,14,15$, 16]. One notable known design choice is selecting the modulation scheme, since modulation type determines the shape of the temporal waveform, the hardware requirements (for example, the dynamic range of the frontend receiver), the resilience of the channel to interference, and the achievable throughput of the channel. By judicious selection of the modulation type, channel throughput can be maximized, and many research teams have investigated various algorithms and strategies for determining the optimal modulations for both microwave and terahertz wireless links $[17,18,19]$.

Many modulation schemes are possible, and all carry their own benefits and drawbacks. However, the prototype terahertz links in the demonstrations listed earlier em- ploy various orders of quadrature amplitude modulation, collectively known as $M$-QAM schemes, including Binary Phase Shift Keying (BPSK, or 2-QAM), Quadrature Phase Shift Keying (QPSK, or 4-QAM), 8-QAM and 16-QAM. In an $M$-QAM scheme, binary data is encoded as communi- cation symbols, distinct combinations of amplitude and phase of the carrier wave, each of which represent one or more bits of data. The modulation order $M$ specifies how many such combinations of amplitude and phase are recognized by the receiver, and $\log _{2}(M)$ bits of data are carried by each symbol.

As the modulation order of the communication system is increased, each symbol transition carries more information, which consequently increases the spectral efficiency of the link. Spectral efficiency is a measure of how many bits of data are transferred per unit of bandwidth utilized by the communication system, typically given in units of $(\underline{\underline{b} \text { its }}) / \mathrm{Hz}$. While the spectral efficiency realized in a physical communication system depends on many factors (such as the coding scheme, Signal-To-Noise Ratio (SNR), and fading characteristics of the channel), the theoretical maximum spectral efficiency of an $M$-QAM scheme is ultimately given by, and scales with modulation order according to, $\log 2(M)[20]$.
In general, this increase in spectral efficiency makes higher order modulations the most attractive, due to the fact that more information can be sent within in a given bandwidth or, conversely, that the system requires less bandwidth to maintain a given data rate.

However, higher-order modulations are not always viable to use. When a system is constrained to operate below some fixed maximum power, the phase and amplitude of all communication symbols must fall within a finite region of the phase/amplitude plane that satisfies that power constraint. Increasing the number of communication symbols necessarily means that symbols must take on increasingly similar values of amplitude and/or phase, as more symbols have to be placed within the finite region satisfying the power constraint. When the receiver must differentiate between a large number of similar symbols with high resolution, injected noise can easily shift the amplitude and/or phase of the received waveform so that symbols are received in error, much more so than for a lower-order modulation scheme where symbol regions are larger and more widely spaced. As a result, higher-order $M$-QAM schemes have more stringent requirements on the minimum SNR allowed at the receiver for effective operation. Fig. 1 illustrates the increase in SNR required by higher-order $M$-QAM schemes in order to maintain a given bit error rate.

When designing a communication link, the modulation type is chosen so that an acceptable error rate is maintained under the worst-case SNR the link is designed to handle. In order to decrease the outage probability during times when the signal is strongly attenuated, and to increase the capacity when channel conditions are favorable, many communication systems employ optimization algorithms that actively select the order of the modulation scheme used [17]. These optimization routines switch between modulation orders as channel conditions vary, such that the resulting link is both more reliable (in terms of outage probability) and operates with a higher average capacity.

Terahertz links will, of course, likewise benefit from these type of optimization routines $[18,17]$, whether the bandwidth is occupied by a single link, or filled with a large number of subcarriers [21]. However, due to the huge bandwidths available for terahertz communication links, and the high frequencies at which they operate, the optimal modulation type will not be determined by SNR (that is, fading) alone. Our work indicates that the Group Velocity Dispersion (GVD) caused by molecular resonances in the atmosphere can result in counter-intuitive behavior over the lower terahertz bands, in which the severity of Inter-Symbol Interference (ISI) depends not only on band- width (as expected), but also on the modulation type used, even in the absence of noise. 


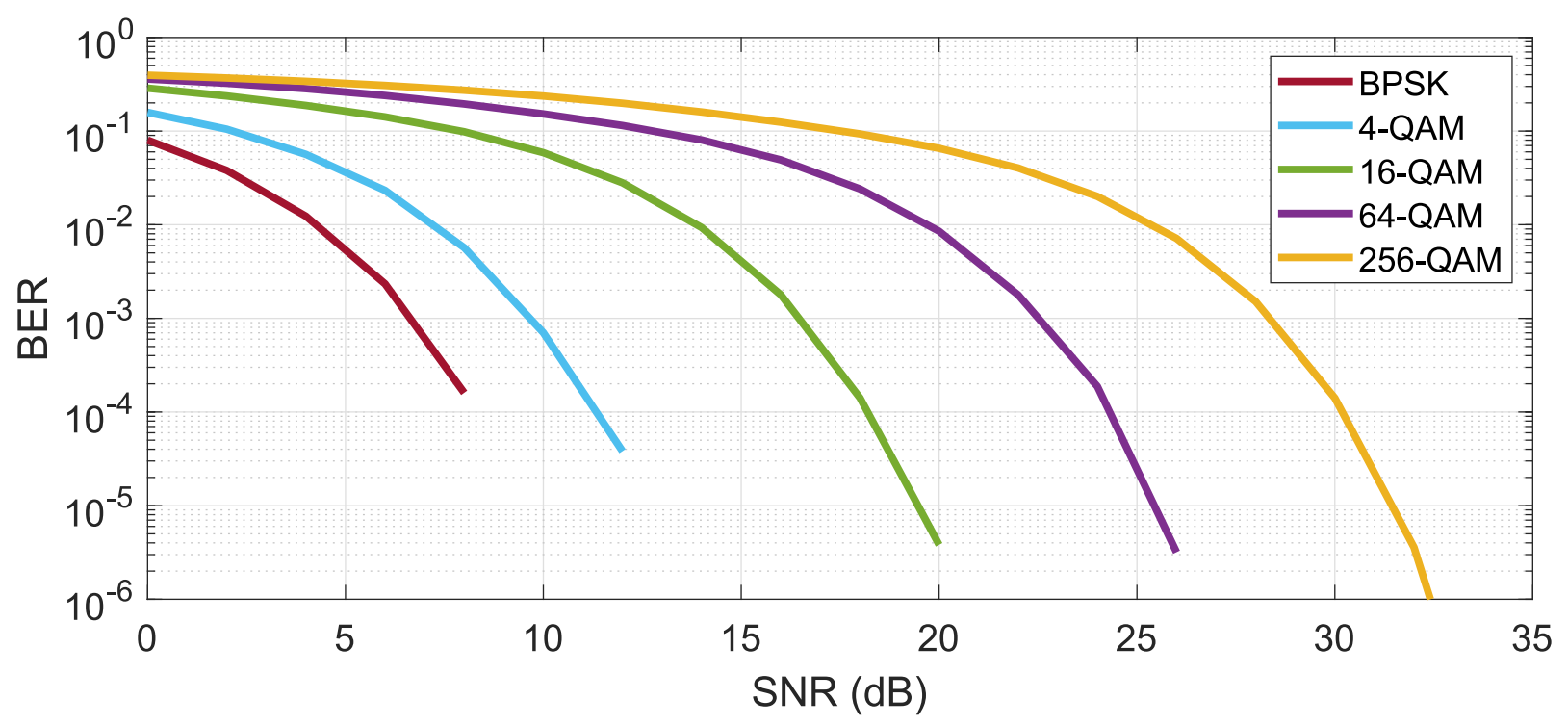

Fig. 1 - Bit error rate versus SNR "waterfall” plots for an $M$-QAM communication system, with $M=2$, 4, 16, 64, and 256. Higher-order modulations have closer symbol spacing under equivalent power requirements, resulting in a higher SNR required for equivalent error performance to a lower order modulation, assuming the absence of group velocity dispersion.

\section{METHODOLOGY}

In order to quantitatively measure the impact of ISI caused by atmospheric GVD, bit error rate simulations were performed using a channel model founded upon an accurate understanding of atmospheric molecular resonances. It is from this atmospheric model that all the effects accounted for in this work were derived. Specifically, the channel considered in this study was a Linear Time-Invariant (LTI) channel with Additive White Gaussian Noise (AWGN) and no obstruction, multipath propagation, or Doppler effects. However, the transfer function of the atmosphere itself was modeled as variable over frequency in both absorption and refractive index, which gives rise to the behavior observed in our results. Even though our assumption of an LTI AWGN channel is much simpler than the environments usually encountered by wireless link designers at terahertz frequencies, the fact that our results arise from the properties of the atmosphere rather than complex and situation-specific channel effects make them applicable to a wide range of channels, including those significantly more complex that that presented here [22].

The atmospheric transfer function is described most generally as $H_{a}(\omega)=\alpha(\omega) \exp [-j \phi(\omega)]$, where $\alpha(\omega)$ and $\phi(\omega)$ are the frequency-dependent attenuation and phase shift imparted by the atmosphere, respectively, and $j=\sqrt{-1}$. This non-unity transfer function arises from the interaction of various atmospheric gas species with terahertz-frequency radiation. Most notable among these are water vapor and diatomic oxygen, which exhibit strong rotational and vibrational resonances within and above the terahertz bands. While the amplitude (absorption) term of $H_{a}(\omega)$ is most often discussed, the phase term $\phi(\omega)$ is equally important to propagation, and to- gether these terms determine the complex index of refraction of the atmosphere. This complex index is obtained by a combination of Molecular Response Theory (MRT) [23] and continuum effects $[24,25]$, in which the broadened absorption lines of all the $\mathrm{H}_{2} \mathrm{O}$ and $\mathrm{O}_{2}$ molecular resonances from 0 to $5 \mathrm{THz}$ are found by MRT, summed, then added to the continuum absorption. This has been shown to accurately model atmospheric behavior over the subterahertz bands, and accounts for the contribution of all relevant molecular resonances up to $5 \mathrm{THz}$.

In addition to the atmospheric effects, pulse shaping filters also shape the transmitted waveform, limit the bandwidth of the signal, and reduce ISI. In our simulations, a raised cosine filter $H_{r c}(\omega)$ with a roll-off factor of 1 was used, and incorporated into the channel model by applying it directly to the atmospheric transfer function in frequency domain, yielding a channel transfer function $H_{c}(\omega)=H_{r c}(\omega) \times H_{a}(\omega)$. The impulse response of the complex channel transfer function can then be derived as $h_{c}(t)=\mathcal{F}^{-1}\left[H_{c}(\omega)\right]$, where $\mathcal{F}^{-1}$ indicates the inverse Fourier transform.

Once the impulse response of the channel is known, a data vector containing complex valued communication symbols is generated. The symbols in the data stream occur with equal distribution, but the data stream is not completely random. Rather, it is generated such that combinations of symbols are also equally distributed, so every possible permutation of $k$ symbols occurs an equal number of times for a specified $k$. This is necessary because the severity of ISI experienced by a communication symbol depends on the value and order of the neighboring symbols, not on the value of the symbol itself. This data stream is convolved with the channel impulse response, resulting in a sequence of 
non-ideal communication symbols in the time domain, which have experienced a nonlinear phase shift due to GVD in the atmosphere, resulting in ISI in the time domain. At this point, AWGN is added to the distorted symbol sequence, which is then demodulated and compared to the original symbol vector to determine the number of errors. This procedure is repeated until enough iterations have run to ensure the errors generated are true to the stochastic distribution of the noise. Averaging the error rates observed on each iteration gives the error rate of the link for that particular combination of distance, bandwidth, modulation type, and atmospheric properties.

This simulation process has the advantage of abstracting away much of the hardware, focusing on the mathematical, fundamental interactions between the data-carrying symbols and the atmospheric channel. Notably, it also defines parameters such as received SNR directly prior to demodulation, so that the results are applicable to a broad range of physical systems. For a much more in-depth description of the simulation process using a slightly different mathematical convention, readers are referred to our previous work [26], which is currently under consideration for publication at the time of this writing.

\section{RESULTS AND DISCUSSION}

A principal result taken from our simulations is that GVD can produce increasing SER in two opposing cases: when low bandwidth and high order modulation is employed and when high bandwidth and low order modulation is employed. Moreover, there exists an optimum tradeoff between modulation order and bandwidth that minimizes SER due to GVD for a particular desired data rate. In order to clarify this point, we have chosen to conduct our simulations such that data rate is held constant, whereas bandwidth and modulation order are variable. Further justification for this approach is offered later in the discussion. To elaborate on our results, the following relationships between modulation order and GVD were found:

For a high bandwidth link with low modulation order, achievable data rate is high, but the link suffers a high number of symbol errors because of the large frequencydependent change in refractive index across the bandwidth (high GVD) causes severe ISI, large enough to push received symbols across the broadly-spaced decision boundaries used in low-order modulation types.

For a low bandwidth link with high order modulation, the achievable data rate is equally high, but the link still suffers a high number of symbol errors due to dispersion. This time, the errors are not because of a large frequency dependency in the narrow channel, but because decision boundaries are so tightly spaced on the constellation diagram that even the small amount of GVD exhibited by the channel is enough to push received symbol values across them, again causing ISI.
A compromise between these two extremes allows for high bit rates with higher dispersion tolerance when bandwidth and modulation order are properly balanced. Note that the remaining combinations of bandwidth and modulation order fare quite poorly: a high bandwidth link with high order modulation can have a very high capacity but suffers severe ISI due to simultaneously high dispersion and tight decision boundaries, while a low band-width link with low modulation order has greatly reduced data capacity, defeating the purpose of terahertz commu- nications. An example of the simulation results that led us to these conclusions is illustrated in Fig. 2, which shows the bit-error rate of a $60 \mathrm{~Gb} / \mathrm{s} M-\mathrm{QAM}$ communication link for five different modulation orders over 0 to $20 \mathrm{~km}$.

It is important to note that atmospheric dispersion is a cumulative phenomenon, meaning the greater distance a signal propagates, the more dispersion accumulates and affects that signal. It is also important to note that no noise is added to the signal in Fig. 2, which allows us to confidently state that any change in error rates observed are due to GVD increasing with distance, and the that differences between the various curves are due to changes in modulation order (which affects both bandwidth and symbol spacing on the constellation diagram). Even in the absence of noise, increasing dispersion over distance will eventually cause some communication symbols to be misinterpreted by the receiver for all modulation types, resulting in a bit error rate that rises rapidly from insignificance to some finite value, often by several orders of magnitude in only a kilometer or two.

The point at which the error rate jumps from insignificance to a finite value is the uncompensated "dispersion limit" of that link, marked by vertical dashed lines in Fig. 2. Beyond this limit, the error rate of the link cannot be continuously improved by increasing the SNR, because dispersion is the dominant source of errors [26]. For the 60 $\mathrm{Gb} / \mathrm{s}$ link shown in Fig. 2, BPSK has the lowest dispersion limit, meaning it is most severely affected by atmospheric temporal dispersion. 4-QAM, or QPSK, is next, followed by 256-QAM, 16-QAM, and finally 64-QAM. In other words, the dispersion limit increases with modulation order for most of the modulation orders simulated, meaning there is more robust operation as the bandwidth decreases.

The results and discussion presented so far may seem obvious and well-established. It is well-known that decreasing the bandwidth of a wireless link operating in a frequency-selective environment will increase the performance of the link by "flattening" the fading profile of the channel, thereby reducing errors, ISI, and the complexity of signal processing. The atmosphere is a frequencyselective channel over the huge bandwidths available to terahertz communication links, so it may not initially seem surprising that as we decrease the bandwidth we also observe an improvement in error rate. However, closer inspection of the data reveal additional and unexpected behaviors that are not readily explained 


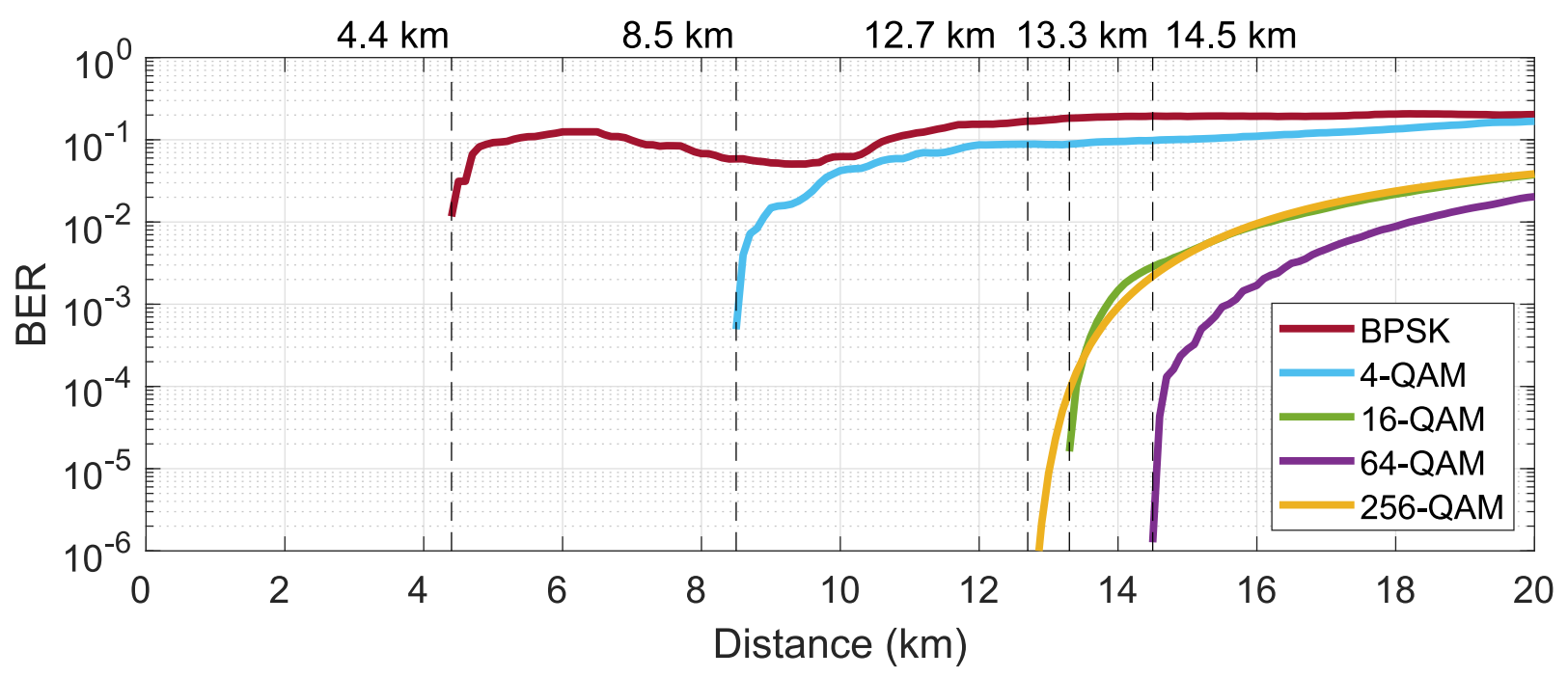

Fig. 2 - Bit error rate versus distance "reverse waterfall” plot for a noiseless $60 \mathrm{~Gb} / \mathrm{s}$ link, centered at $250 \mathrm{GHz}$. Dashed vertical lines mark "dispersion limits," the distance at which uncompensated dispersion begins to deterministically cause bit errors, which cannot be overcome by increasing the SNR. Atmospheric conditions are water vapor density $\rho_{\mathrm{wv}}=10.37 \mathrm{~g} / \mathrm{m}^{3}\left(60 \%\right.$ relative humidity at $\left.20^{\circ} \mathrm{C}\right)$. The decrease in the error rate of the BPSK curve over $7 \mathrm{~km}$ to $10 \mathrm{~km}$ is a consequence of how atmospheric GVD shifts the received value of communication symbols. Because BPSK modulates only a single dimension in the complex symbol space, it experiences fewer errors over the $7 \mathrm{~km}$ to $10 \mathrm{~km}$ region, where dispersion tends to shift a majority of symbols orthagonally to the dimension of modulation. This effect is not strongly observed in higher-order modulations due to their use of the orthagonal (quadrature) dimension, though this effect also produces a slight dip at about $9 \mathrm{~km}$ for the 4-QAM link.

by the usual intuition about wireless systems. If frequency-selective fading was the driver of the increase in error rate, then we would expect to see the error rate improve with every decrease in bandwidth, but the exact opposite is observed for the transition from 64-QAM to 256-QAM. In fact, 256-QAM has a similar dispersion limit to 16-QAM, despite having twice the spectral efficiency, that is, half the bandwidth. The cause for this reversal is that the symbols in 256-QAM are so closely spaced that only a small amount of dispersion is enough to shift them across the decision boundaries and produce errors, even though the frequency-dependent fading due to the atmosphere is essentially flat across the bandwidth. In other words, in the presence of GVD, the decrease in symbol spacing outweighs the decrease in bandwidth due to spectral efficiency gains, resulting in more errors.

This demonstrates that GVD, not frequency-selective fading, is responsible for these errors. It is worth remembering that the results shown in Fig. 2 are for a single link, with no multipath interference, over an LTI channel with no noise added. The errors observed are solely due to the frequency dependent refractive index of the atmosphere. Consequently, these results show that in the terahertz and sub-terahertz bands, reducing bandwidth does not necessarily improve error rate performance because the shape of the waveform (that is, modulation type) also matters, due to the atmospheric interaction. This is a counter-intuitive result that is uniquely different from free-space microwave links.

While the noiseless case is instructive, it is not always rep- resentative of the real world. Fig. 3 shows the error per-formance of the 4-QAM and 16-QAM links of Fig. 2 in the presence of varying amounts of noise, as described in the figure caption. As expected, poor SNR impacts the error rate of the high-order 16-QAM link more severely than the 4-QAM link. It is significant to note that at the lower order 4-QAM link, the error rate can be improved in some cir-cumstances, even with poor SNR, by shifting to a higher or- der modulation, whenever the cost of degraded SNR performance is offset by reduced GVD in the more spectrally efficient modulation.

For example, examine the 4-QAM link operating at 8 kilometers with an SNR of $20 \mathrm{~dB}$, denoted by the point ' $a$ ' called out on the plot. The expected uncompensated error rate is $0.59 \%$. While it may be intuitive to decrease the modulation order to improve the error performance, the results in Fig. 2 show this is not advisable; a BPSK link under the same conditions is operating beyond the dispersion limit, and has a high error rate of about 7.5\%, even in the effective total absence of noise. Rather, if the modulation order is increased to 16, then Fig. 3 shows the error rate is decreased to $0.035 \%$ for the same SNR of $20 \mathrm{~dB}$, more than an order of magnitude improvement (denoted by the point ' $b$ ' called out on the plot). In fact, the results presented in Fig. 3 show that when the link distance is above $8.5 \mathrm{~km}$ (shown by the vertical dashed line) and the SNR greater than $20 \mathrm{~dB}$, switching from 4-QAM to 16-QAM will always improve the error rate, due to the greater spectral efficiency (and thus lower bandwidth and GVD) of the 16-QAM scheme.

For a second illustration, now consider the 16-QAM link operating at $14 \mathrm{~km}$ in Fig. 4, with a high SNR of $40 \mathrm{~dB}$. The expected error rate is $0.148 \%$ (denoted by point ' $a$ ' on the plot). To improve this error rate, the default choice might be to decrease the modulation order, but again, this worsens the error rate to a value of $9.5 \%$ for $4-\mathrm{QAM}$ 


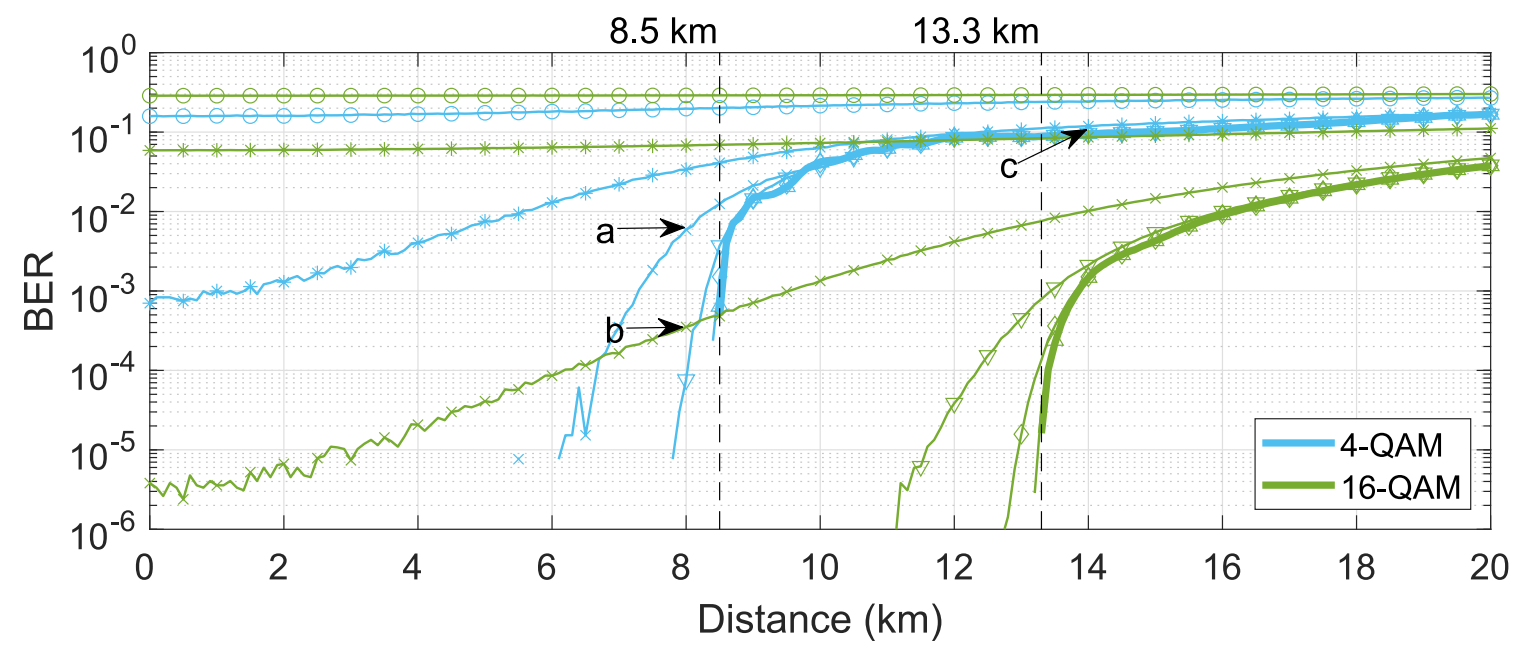

Fig. 3 - Reverse waterfall plot for $M$-QAM links, $M=4,16$, over distances from 0 to $20 \mathrm{~km}$ with various SNRs. SNR values of 0 , 10 , 20, 30, 40, and $50 \mathrm{~dB}$ are designated by markers $\bigcirc, *, \times, \nabla, \diamond$, and $\triangle$ respectively, and infinite SNR is denoted by a thick, solid line with no marker. Atmospheric conditions are the same as in Fig. 2. The fact that the $40 \mathrm{~dB}, 50 \mathrm{~dB}$, and infinite SNR curves are almost indistinguishable because beyond $40 \mathrm{~dB}$, dispersion is the dominant source of errors, rather than noise.

(according to point ' $c$ ' on Fig. 3). However, increasing the modulation order to 64 offers improvement according to Fig. 4, reducing the error rate to $4.7 \times 10^{-5}$ ( point ' $b$ ' on the plot). In this case, the improvement again relies on the increased spectral efficiency of the higher order modulation scheme, with the stipulation that the SNR be $40 \mathrm{~dB}$ or greater. This exacting constraint on SNR arises from the small spacing between symbol decision boundaries for the higher-order link, made even stricter by the fact that dispersion, though reduced, has still shifted some of the received symbols closer to the decision boundaries.

Thirdly, notice from Fig. 2 that, for the $60 \mathrm{~Gb} / \mathrm{s}$ case presented here, there are some modulation schemes that, in general, constitute poor choices for a link without dispersion compensation. Namely, a 256-QAM scheme has a worse error rate at all distances and SNRs than either 16-QAM or 64-QAM (with the exception of a slight and insignificant region around $14 \mathrm{~km}$ in the noise-free case, where it has performance marginally better than the 16-QAM scheme). While BPSK and 4-QAM do underperform 256-QAM over long distances with higher SNR, there are also two other modulation types (16-QAM and 64-QAM) that outperform 256-QAM in nearly all situations, so while 256-QAM is an improvement over some modulation schemes, it is never the best choice (and this holds true for all higher SNRs as well).

At this point in the discussion, there are a few assumptions that need to be addressed. One point of concern may be that in most applications, the bandwidth of a wireless link is fixed and the data rate varies with modulation order, while in the results we present, the band-width varies with modulation order while data rate is held constant. However, we are not proposing this is how future terahertz links should operate; indeed, variable bandwidth channels would be both an engineering and a regulatory challenge, and probably are not appropriate for most circumstances. Rather, we chose to present our data in this manner because allowing bandwidth to vary with modulation order makes the counter-intuitive behavior of the spectrallyefficient, low bandwidth links (that is, 256-QAM) the most clear and explicit. This does not change our simulation results; it is just a data-presentation choice. Varying the bandwidth of the link was the best way to show that decreasing the bandwidth does not necessarily decrease the error rate, and that modulation type becomes an important factor due to atmospheric GVD.

Finally, all discussion up until this point has been focused on communication links in which dispersion is uncompen- sated. Though GVD has not historically been a concern for wireless communication systems (owing to the com- paratively narrow bandwidth of legacy microwave com- munication links), it has been extensively investigated in fiber optics, where dispersion-compensating technology is relatively mature. Additionally, there are other forms of temporal dispersion that have been identified, studied, and compensated in existing wireless links, which often arise from multipath propagation. Although atmospheric GVD is a new phenomena for wireless links, dispersion in general is not. This may lead some to think that since dispersion can and has been compensated by both photonic [27] and electronic [28, 29] means, then these technologies would be readily adapted for use in a terahertz wireless communication system. Specifically, it might be assumed digital signal processing filters, also known as equalizers, will be able to compensate GVD and thus render the problem of GVD irrelevant. 


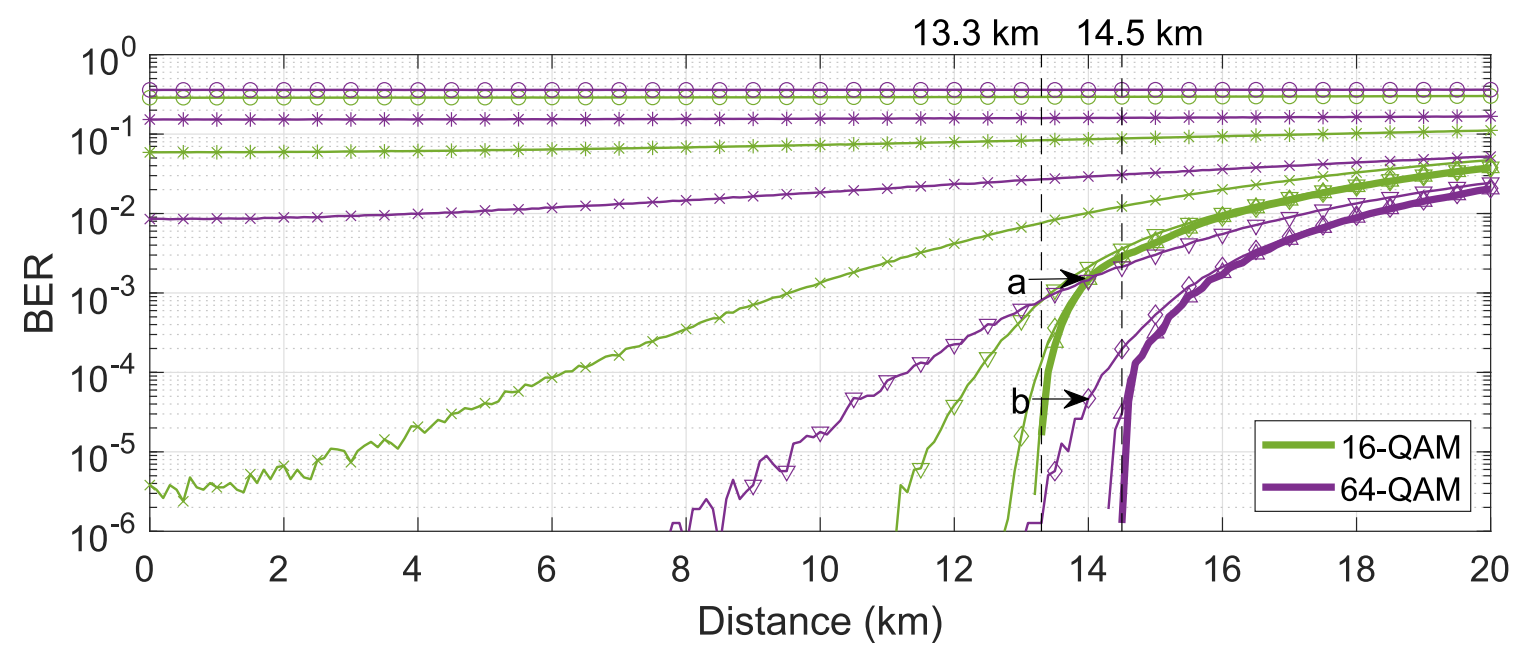

Fig. 4 - Reverse waterfall plot for $M$-QAM links, $M=16,64$, over distances from 0 to $20 \mathrm{~km}$ with various SNRs. SNR values of $0,10,20,30$, 40 , and $50 \mathrm{~dB}$ are designated by markers $\bigcirc, *, \times, \nabla, \diamond$, and $\triangle$ respectively, and infinite SNR is denoted by a thick, solid line with no marker. Atmospheric conditions are the same as in Fig. 2.

While equalizers are certainly theoretically capable of compensating dispersion, whether they will be physically realizable for terahertz frequencies (and, if so, when) is still yet to be determined. There are still questions that remain to be answered before we can confidently assert which equalizer architectures will be most suited to operation in the terahertz bands. In $4 \mathrm{G}$ architectures utilizing orthogonal frequency division multiplexing, equalizers operate on channels at most $20 \mathrm{MHz}$ wide, and this is the dominant wireless technology. However, in the terahertz bands, the signal bandwidth may be up to $100 \mathrm{GHz}$, potentially over three orders of magnitude larger! Even the fiber optic equalizers referenced previously typically have bandwidths less than $100 \mathrm{GHz}$ [30]. This high bandwidth significantly complicates filter design. If terahertz sub-bands are kept only a few tens of kilohertz wide in order to avoid this problem, then the number of sub-bands (and thus equalizers) scales up by potentially four orders of magnitude. Further complications include 10 to 100 times greater Doppler shifts, noise bandwidths two to three orders of magnitude larger, and dispersion profiles that change with weather, not to mention the is- sues of receiver linearity, phase noise, and dynamic range which are already challenges for $3 \mathrm{G}$ and $4 \mathrm{G}$ hardware [31]. While none of this changes the fact that dispersion is theoretically reversible, it does raise the question: are current equalization algorithms and the digital hardware on which they are implemented capable of performing the task? Current research is presently being undertaken to investigate these issues [32], and bottlenecks related to sampling rate and signal processing limitations have been identified [33, 34]. Presently, it seems premature to assume that the equalization and signal processing technologies we currently have will carry over to terahertz channels without significant modification and innovation.
Accordingly, we have not included equalization routines in our simulations for concern they would produce results that are not necessarily realistic. Furthermore, we wish to limit the scope of this paper to a characterization and description of the GVD induced by the atmosphere, and its interaction with modulation type. If and when dispersion compensating technology is implemented in future terahertz communication systems, the judicious selection of modulation type will reduce the performance requirements placed on such technology by utilizing modulation schemes naturally resistant to dispersion-induced bit errors. This could be especially important for relaxing the signal processing burden in terahertz transceivers.

\section{CONCLUSION}

In this work, we leveraged highly accurate models of the atmosphere to predict the effects of uncompensated atmospheric GVD on the bit error rate of high-capacity terahertz links using various orders of $M$-QAM. A significant finding was that, due to GVD, unintuitive situations arise in which higher-order modulations offer superior error rate performance than lower-order modulations. This is contrary to what would be expected in a traditional wireless link with a lower bandwidth, in which the selection of modulation type is dominated by the SNR alone. It is anticipated that this will need to be taken into account by both future link designers and adaptive modulation algorithms attempting to select the ideal modulation scheme for present channel conditions. A related finding was that, in uncompensated links, there are some modulation orders that should not be used (or are at least never the best choice). Specifically, high-order modulations, such as 256-QAM (and above) suffer from stringent requirements on both SNR and maximum allowable symbol shift due to dispersion, which when combined lead to suboptimal performance for all or nearly all combinations of links distance and SNR. 


\section{ACKNOWLEDGMENT}

This material is based upon work supported by the National Science Foundation Graduate Research Fellowship Program under Grant No. 1746055 and by the National Science Foundation under Grant No. 2018110. Any opinions, findings, and conclusions or recommendations ex- pressed in this material are those of the author(s) and do not necessarily reflect the views of the National Science Foundation.

\section{REFERENCES}

[1] Walid Saad, Mehdi Bennis, and Mingzhe Chen. "A vision of 6G wireless systems: Applications, trends, technologies, and open research problems". In: IEEE network 34.3 (2019), pp. 134-142.

[2] GMDT Forecast. "Cisco visual networking index: global mobile data traffic forecast update, 20172022". In: Update 2017 (2019), p. 2022.

[3] L. Chettri and R. Bera. "A Comprehensive Survey on Internet of Things (IoT) Toward 5G Wireless Systems". In: IEEE Internet of Things Journal 7.1 (2020), pp. 16-32. DOI: 10 . 1109 / JIOT . 2019 . 2948888.

[4] K. Shafique, B. A. Khawaja, F. Sabir, S. Qazi, and M. Mustaqim. "Internet of Things (IoT) for Next-Generation Smart Systems: A Review of Current Challenges, Future Trends and Prospects for Emerging 5G-IoT Scenarios". In: IEEE Access 8 (2020), pp. 23022-23040. DOI: 10 . 1109/ ACCESS . 2020.2970118.

[5] Y. Xing and T. S. Rappaport. "Propagation Measurement System and Approach at $140 \mathrm{GHz}$-Moving to 6G and Above $100 \mathrm{GHz}$. In: 2018 IEEE Global Communications Conference (GLOBECOM). 2018, pp. 16. DOI: 10.1109/GLOCOM. 2018.8647921.

[6] Ho-Jin Song and Tadao Nagatsuma. "Present and future of terahertz communications". In: IEEE transactions on terahertz science and technology 1.1 (2011), pp. 256-263.

[7] A Hirata, T Kosugi, H Takahashi, J Takeuchi, K Murata, N Kukutsu, Y Kado, S Okabe, T Ikeda, F Suginosita, et al. "5.8-km 10-Gbps data transmission over a 120-GHz-band wireless link". In: 2010 IEEE International Conference on Wireless Information Technology and Systems. IEEE. 2010, pp. 1-4.

[8] Hiroyuki Takahashi, Toshihiko Kosugi, Akihiko Hirata, Jun Takeuchi, Koichi Murata, and Naoya Kukutsu. "120-GHz-band fully integrated wireless link using QSPK for realtime 10-Gbit/s transmission". In: IEEE transactions on microwave theory and techniques 61.12 (2013), pp. 4745-4753.
[9] Cheng Wang, Changxing Lin, Qi Chen, Bin Lu, Xianjin Deng, and Jian Zhang. "A 10-Gbit/s wireless communication link using 16-QAM modulation in 140-GHz band". In: IEEE Transactions on Microwave Theory and Techniques 61.7 (2013), pp. 2737-2746.

[10] Xinying Li, Jianjun Yu, Kaihui Wang, Yuming Xu, Long Chen, Li Zhao, and Wen Zhou. "Bidirectional delivery of 54-Gbps 8QAM $\mathrm{W}$-band signal and 32-Gbps 16QAM K-band signal over 20-km SMF-28 and 2500-m wireless distance". In: Optical Fiber Communication Conference. Optical Society of America. 2017, Th5A-7.

[11] Ingmar Kallfass, Jochen Antes, Axel Tessmann, Thomas Zwick, and Ralf Henneberger. "Multi-Gigabit high-range fixed wireless links at high millimeterwave carrier frequencies". In: 2017 IEEE Radio and Wireless Symposium (RWS). IEEE. 2017, pp. 45-48.

[12] Qiuyu Wu, Changxing Lin, Bin Lu, Li Miao, Xin Hao, Zhaohui Wang, Yi Jiang, Wenqiang Lei, Xianjing Den, Hongbin Chen, et al. "A $21 \mathrm{~km} 5$ Gbps real time wireless communication system at 0.14 THz". In: 2017 42nd International Conference on In-frared, Millimeter, and Terahertz Waves (IRMMW-THz). IEEE. 2017, pp. 1-2.

[13] M. Polese, J. M. Jornet, T. Melodia, and M. Zorzi. "Toward End-to-End, Full-Stack 6G Terahertz Networks". In: IEEE Communications Magazine 58.11 (2020), pp. 48-54. DOI: 10 . 1109 / MCOM . 001.2000224.

[14] S. Ghafoor, N. Boujnah, M. H. Rehmani, and A. Davy. "MAC Protocols for Terahertz Communication: A Comprehensive Survey". In: IEEE Communications Surveys Tutorials 22.4 (2020), pp. 2236-2282. DoI: 10.1109/COMST . 2020.3017393.

[15] T. Schneider, A. Wiatrek, S. Preussler, M. Grigat, and R. Braun. "Link Budget Analysis for Terahertz Fixed Wireless Links". In: IEEE Transactions on Terahertz Science and Technology 2.2 (2012), pp. 250-256. DoI: 10.1109/TTHZ.2011.2182118.

[16] K. Rikkinen, P. Kyosti, M. E. Leinonen, M. Berg, and A. Parssinen. "THz Radio Communication: Link Budget Analysis toward 6G". In: IEEE Communica- tions Magazine 58.11 (2020), pp. 22-27. DOI: 10 . 1109/MCOM.001.2000310.

[17] A. A. Boulogeorgos, E. N. Papasotiriou, and A. Alexiou. "A Distance and Bandwidth Dependent Adaptive Modulation Scheme for $\mathrm{THz}$ Communications". In: 2018 IEEE 19th International Workshop on Signal Processing Advances in Wireless Communications (SPAWC). 2018, pp. 1-5. DoI: 10.1109/SPAWC. 2018.8445864 . 
[18] C. Han and I. F. Akyildiz. "Distance-Aware Bandwidth-Adaptive Resource Allocation for Wireless Systems in the Terahertz Band". In: IEEE Transactions on Terahertz Science and Technology 6.4 (2016), pp. 541-553. DoI: 10 . 1109 / TTHZ . 2016.2569460.

[19] C. Han and I. F. Akyildiz. "Distance-aware multicarrier (DAMC) modulation in Terahertz Band communication". In: 2014 IEEE International Confe-rence on Communications (ICC). 2014, pp. 5461-5467. DOI: 10.1109/ICC. 2014.6884190.

[20] Pei Yang, Zeliang Ou, and Hongwen Yang. "Capacity of AWGN and Rayleigh Fading Channels with $M$-ary Inputs". In: 2018 IEEE 29th Annual International Symposium on Personal, Indoor and Mobile Radio Communications (PIMRC). IEEE. 2018, pp. 1-6.

[21] Shi Jia, Xianbin $\mathrm{Yu}, \mathrm{Hao} \mathrm{Hu}$, Jinlong $\mathrm{Yu}$, Pengyu Guan, Francesco Da Ros, Michael Galili, Toshio Morioka, and Leif K. Oxenløwe. "THz photonic wireless links with 16-QAM modulation in the 375$450 \mathrm{GHz}$ band". In: Opt. Express 24.21 (Oct. 2016), pp. 23777-23783. DOI: 10.1364/ OE. 24.023777. URL: http : / / wWW . opticsexpress . org /abstract.cfm? URI $=o e-24-21-23777$.

[22] Chong Han and Yi Chen. "Propagation Modeling for Wireless Communications in the Terahertz Band". In: IEEE Communications Magazine 56.6 (2018), pp. 96-101. DOI: 10.1109/ MCOM. 2018. 1700898.

[23] Yihong Yang, Mahboubeh Mandehgar, and Daniel R. Grischkowsky. "Understanding THz Pulse Propagation in the Atmosphere". In: IEEE Transactions on Terahertz Science and Technology 2.4 (2012), pp. 406-415. DOI: 10.1109/TTHZ.2012.2203429.

[24] John F O’Hara and Daniel R Grischkowsky. “Comment on the veracity of the ITU-R recommendation for atmospheric attenuation at terahertz frequencies". In: IEEE Transactions on Terahertz Science and Technology 8.3 (2018), pp. 372-375.

[25] Yihong Yang, Mahboubeh Mandehgar, and D Grischkowsky. "Determination of the water vapor continuum absorption by THz-TDS and Molecular Response Theory". In: Optics Express 22.4 (2014), pp. 4388-4403.

[26] Karl Strecker, Sabit Ekin, and John OHara. Fundamental Performance Limits on Terahertz Wireless Links Imposed by Group Velocity Dispersion. 2021. arXiv: 2104.00611 [eess.SP].
[27] Karl Strecker, Sabit Ekin, and John F O’Hara. “Compensating atmospheric channel dispersion for terahertz wireless communication". In: Scienti. ic reports 10.1 (2020), pp. 1-8. DOI: 10.1038/s41598020-62692-7.

[28] Julián S Bruno, Vicenç Almenar, Javier Valls, and Juan L Corral. "Real-time $20.37 \mathrm{~Gb} / \mathrm{s}$ optical OFDM receiver for PON IM/DD systems". In: Optics express 26.15 (2018), pp. 18817-18831.

[29] Amit Raikar, Amol Jirage, and Ashwini Narake. "A SURVEY: DISPERSION COMPENSATION TECHNIQUES FOR OPTICAL FIBER COMMUNICATION". In: International Journal 15 (2019), 16th.

[30] Xing Ouyang, Giuseppe Tall, Mark Power, and Paul Townsend. "Experimental demonstration of 112 Gbit/s orthogonal chirp-division multiplexing based on digital up-conversion for IM/DD systems with improved resilience to system impairments". In: 2018 European Conference on Optical Communication (ECOC). IEEE. 2018, pp. 1-3.

[31] Somayeh Mohammady, Ronan Farrell, David Malone, and John Dooley. "Performance investigation of peak shrinking and interpolating the PAPR reduction technique for LTE-advance and $5 \mathrm{G}$ signals". In: Information 11.1 (2020), p. 20.

[32] Priyangshu Sen, Dimitris A. Pados, Stella N. Batalama, Erik Einarsson, Jonathan P. Bird, and Josep M. Jornet. "The TeraNova platform: An integrated testbed for ultra-broadband wireless communications at true Terahertz frequencies". In: Computer Networks 179 (2020), p. 107370. ISSN: 1389-1286. DoI: https : / / doi . org / 10 . 1016 / j . comnet . 2020 . 107370. URL: https : / / www . sciencedirect . com / science / article / pii /S1389128620304473.

[33] Viduneth Ariyarathna, Arjuna Madanayake, and Josep Miquel Jornet. "Real-Time Digital Baseband System for Ultra-Broadband THz Communication". In: 2020 45th International Conference on Infrared, Millimeter, and Terahertz Waves (IRMMW-THz). 2020, pp. 1-2. DoI: 10 . 1109 / IRMMW - THz46771. 2020.9370733.

[34] Hadi Sarieddeen, Mohamed-Slim Alouini, and Tareq Y Al-Naffouri. "An overview of signal processing techniques for terahertz communications". In: arXiv preprint arXiv:2005.13176 (2020). 


\section{AUTHORS}

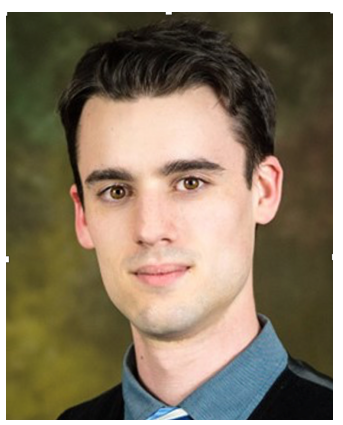

Karl L. Strecker received his B.S. in 2018 and M.S. in 2020, both in electrical engineering from Oklahoma State University. $\mathrm{He}$ is currently pursuing his Ph.D. degree in electrical engineering at the same institution. From 2018 to the present, he has worked as a research assistant in the Ultrafast Terahertz and Optoelectronics Laboratory at Oklahoma State University. His research interests include wireless communications, group velocity dispersion management, and terahertz material characterization. Mr. Strecker was a recipient of the 2020 National Science Foundation Graduate Research Fellowship.

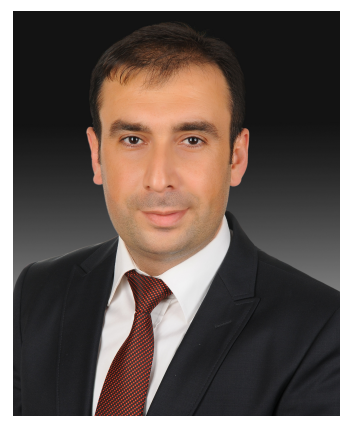

Sabit Ekin received a B.Sc. degree in electrical and electronics engineering from Eskişehir Osmangazi University, Turkey, in 2006, an M.Sc. degree in electrical engineering from New Mexico Tech, Socorro, NM, USA, in 2008, and a Ph.D. degree in electrical and computer engineering from Texas A\&M University, College Station, TX, USA, in 2012.

He was a visiting research assistant with the Electrical and Computer Engineering Program, Texas A\&M University at Qatar from 2008 to 2009. In summer 2012, he was with the Femtocell Interference Management Team in the Corporate Research and Development, New Jersey Research Center, Qualcomm Inc. He joined the School of Electrical and Computer Engineering, Oklahoma State University, Stillwater, OK, USA, as an assistant professor, in 2016. He has four years of industrial experience from Qualcomm Inc., as a Senior Modem Systems Engineer with the Department of Qualcomm Mobile Computing.
At Qualcomm Inc., he has received numerous Qualstar awards for his achievements/contributions on cellular modem receiver design. His research interests include the design and performance analysis of wireless communications systems in both theoretical and practical point of views, interference modeling, management and optimization in 5G, mmWave, HetNets, cognitive radio systems and applications, satellite communications, visible light sensing, communications and applications, RF channel modeling, non-contact health monitoring, and Internet of Things applications.

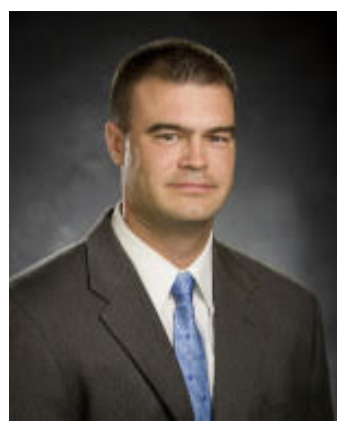

John F. O'Hara (M'05 - SM'19) received his BSEE degree from the University of Michigan in 1998 and his Ph.D. (electrical en-gineering) from Oklahoma State University (OSU) in 2003. Hewas a Director of Central Intel-ligence Postdoctoral Fellow at Los Alamos National Laboratory(LANL) until 2006.

From 2006-2011 he was with the Center for Integrated Nanotechnologies (LANL) and worked on numerous metamaterial projects involving dynamic control over chirality, resonance frequency, polarization, and modulation of terahertz waves. In 2011, he founded a consulting/research company, Wavetech, LLC specializing in automation and IoT devices. In 2017 he joined OSU as an assistant professor in the School of Electrical \& Computer Engineering. His current research involves terahertz wireless communications, terahertz sensing and imaging with metamaterials, IoT, and light-based sensing and communications. He has 3 patents and around 100 publications in journals and conference proceedings. Dr. $\mathrm{O}^{\prime}$ Hara is a Senior Member of IEEE. 